\title{
Micro milling performance assessment of diamond-like carbon coatings on a micro end mill
}

\author{
Tao $\mathrm{Wu}^{*}$ and Kai Cheng \\ Advanced Manufacturing and Enterprise Engineering (AMEE) Department, \\ School of Engineering and Design, Brunel University, Uxbridge, Middlesex UB8 3PH, UK \\ Emails: tao.wu@brunel.ac.uk, kai.cheng@brunel.ac.uk \\ ${ }^{*}$ Corresponding author: Tao Wu
}

\begin{abstract}
In micro milling, unpredictable tool life and premature tool failures are the major constraints for its industrial applications, and prolongation of the tool life so as to enhance the tooling performance presents great challenges. Appropriate coating techniques potentially offer a feasible and promising solution. In the research presented, diamond-like carbon (DLC) films are deposited on a $\varnothing 500 \mu \mathrm{m}$ diameter tungsten carbide (WC) micro end mill by the plasma enhanced chemical vapor deposition (PE-CVD) process. Coating characterization has been undertaken and the DLC coatings are found in good coverage on the tool except for a slight delaminating on the edge corners. Besides, the surface of the amorphous coatings is much smoother than that of WC. In addition, comprehensive cutting performance of the DLC coated tool in dry slot milling of Al 6061-T6 has been compared with those of uncoated tools in both dry and wet conditions. It is observed that the use of DLC coatings can reduce the cutting forces, lessen the tool wear, improve the surface roughness and minimize the microburr formation as compared to the corresponding performance of an uncoated tool in dry cutting. However, the performance improvement is still unreachable to those resulting from the cutting fluid influence.
\end{abstract}

Keywords: micro milling, diamond-like carbon, cutting forces, tool integrity, surface roughness, micro-burr formation, dry cutting

\section{Introduction}

During the last 1-2 decades, micro milling emerges as a fast-growing manufacturing technology with direct, reliable and repeatable capabilities in fabricating miniaturized freeform and high accuracy features or components. However, unpredictable tool life and premature tool failures are the major constraints for industrial applications of the technology. For commercial micro milling tools, their most common material is ultra-fine grain tungsten carbide due to comparably low cost, high hardness, strength and adequate wear resistance characteristics. Theoretically, these tools can be employed in machining almost all engineering materials, but they suffer from severe wear in case of cutting hard materials. In 
order to prolong the tool life so as to improve the tooling performance, hardness, toughness and wear resistance of a tool material must be consistently maintained during machining operations.

Coating techniques potentially offer a promising solution to meet this need. Coatings should be harder than the substrates in order to resist wear, be more inert to resist chemical wear and must adhere well to the substrates [1]. Appropriate coatings can be used on micro milling tools so as to extend their cost effectiveness and efficiency of the production process in terms of improved surface quality, increased tool life span, reduction in machine downtime and reduction in risk of damage as a result of tool wear or breakages [2]. The tool coating also enables the possibility of dry machining due to a lower friction coefficient between the coating and work materials [3].

Aramacharoen et al. [4] reported that AlCrTiN coated micro tools provide better performance in machining hardened die steels in terms of reduced tool wear and improved surface quality as compared to the uncoated ones. In the later research [5], the authors demonstrated that TiN, TiCN, TiAlN, CrN and CrTiAlN coatings help reducing cutting edge chipping and the edge radius wear as well as the micro-burr size.

The most common coatings for commercial micro tools are titanium-based and TiAlN in particular. Although carbide tools with diamond coating layers have recently come on to the market [6], they are not widely employed and are still under research. Diamond coatings for micro mills are advantageous and promising because they are capable of reducing flute clogging by eliminating adhesion of the work material to the tool surface, reducing tool wear due to high hardness and reducing cutting forces due to low friction coefficients against many materials [3].

Torres et al. [7] coated fine-grained diamond (FGD) and nano-crystalline diamond (NCD) films onto tungsten carbide micro end mills by a hot-filament chemical vapour deposition (HF-CVD) process. The performance of both coated tools in dry slot milling of aluminium shows a dramatic improvement in the tool integrity, lower wear rate, no observable adhesion and a significant reduction in the cutting forces compared to those of uncoated ones. However, the diamond coatings experienced severe delaminating due to insufficient bonding strength to the substrate, resulting in catastrophic tool fracture of the cutting edges. Wu et al. [2] applied $2 \mu \mathrm{m}$ NCD thin films on a micro end mill by the similar coating process. Reduced cutting forces, free chip adhesion, lower tool wear, improved surface roughness as well as smaller burrs were achieved in micro milling trials. The authors also demonstrated the possibility of dry cutting by using the NCD coatings on micro tools.

DLC is an amorphous carbon material with a high fraction of $\mathrm{sp}^{3}$ carbon bonding. It possesses many desirable qualities such as high hardness, high optical band gap, high electrical resistivity, chemical inert and anti-adhering, low friction and wear coefficient properties and it can be deposited at substrate temperature lower than $200^{\circ} \mathrm{C}$. Great 
performance improvement has been achieved by applying DLC coatings on conventional cutting tools [8-10]. However, no attempts have been reported on micro tools.

In the research presented in this paper, DLC thin films are applied on the micro end mill in an effort to enhance the tooling performance. Coating characterization is undertaken to evaluate the coating quality. Multiple criteria performance of the DLC coated tool in dry slot milling, including the cutting forces, tool integrity, surface roughness and micro-burr formation are characterized and compared against those of uncoated WC tools in both dry and wet cutting conditions, supported by the analysis and discussions.

\section{DLC films deposition and characterization}

DLC films are deposited on a two-flute end mill in $\varnothing 500 \mu \mathrm{m}$ diameter and $30^{\circ}$ helix angle (Magaforce 8507- $\varnothing 0.5 \mathrm{~mm}$ ). The substrate material is K15 carbide, containing $6.5-7 \%$ cobalt, with micro grain size between $6 \mu \mathrm{m}$ and $8 \mu \mathrm{m}$. Before the coating process, qualitative condition of the tungsten carbide tool is examined using a Zeiss Supra 35VP FE-SEM operated at $6 \mathrm{kV}$ as micro tool inspection and assessment is of great importance for the ultimate coating quality. The SEM images of the uncoated micro tool are shown in Fig. 1, and the cutting edge radius of the tool is measured to be $1.5 \mu \mathrm{m}$.

\subsection{DLC films deposition process}

DLC films are coated by radio frequency plasma enhanced chemical vapour deposition (PE-CVD) process and the reactor and system are shown in Fig. 2. One of the main attributes of this process is that the thermal envelope is at near-room temperature, allowing coatings to be deposited onto thermally sensitive materials.

The precursor gas is a mixture of acetylene, argon and tetramethylsilane (TMS). The ratio of argon to acetylene is adjusted by controlling the flow rate of these gases individually. Prior to deposition, the sample is ultrasonically cleaned in acetone for $15 \mathrm{~min}$, and transferred to vacuum chamber with a background pressure below $8 \times 10^{-5}$ torr. It is then sputter-cleaned for $30 \mathrm{~min}$ with argon at flow rate of $30 \mathrm{sccm}$, pressure of $8 \times 10^{-2}$ torr and a bias voltage of $370 \mathrm{~V}$. Subsequently, the bias voltage is adjusted to $450 \mathrm{~V}$ and an interfacial layer is formed by adjusting argon flow rate to $10 \mathrm{sccm}$, and introducing TMS with flow rate of $25 \mathrm{sccm}$. This layer enhances the adhesion of the film to the carbide substrate. Once the interfacial layer is formed, acetylene gas with flow rate of $60 \mathrm{sccm}$ is introduced into the chamber and the rate of TMS gas flowing into the system is reduced by half to form a transition layer. The argon flow rate and bias are maintained constant for the film deposition process. After 15min, the TMS gas flowing into the system is cut off and the DLC films are deposited from argon diluted acetylene gas at a bias voltage of $450 \mathrm{~V}$. The coating process continues for another $15 \mathrm{~min}$, allowing the DLC films of approximately $2 \mu \mathrm{m}$ in thickness to be produced.

\subsection{Characterization of the DLC films}


Integrity of the DLC coated micro end mill is inspected and analyzed after the coating process and SEM images of the tool together with corresponding coatings are illustrated in Fig.3.

Due to complex geometrical configuration of the micro end mill, it is quite difficult to achieve perfect seeding results. The surface of DLC coatings emerges to be fairly smooth and the amorphous structure films adhere well to the tungsten carbide substrates, with an exception of slight delaminating on the sharp edge corners, as shown in Fig.3 (c). The value of measured coating thickness is around $2 \mu \mathrm{m}$ (see Fig. 3 (d)), which matches the estimated one based on the growth time. The surface roughness, Ra, of DLC coatings on the tool shank is measured using a white light interferometer Zygo NewView 5000. The value is $29 \mathrm{~nm}$ as compared with $78 \mathrm{~nm}$ of an uncoated micro tool, as given in Fig.4.

\section{Micro milling trials}

Micro milling operations are performed on an ultra-precision micro milling machine UltraMill and the experiment has the same setup as in the literatures [11-12]. A dynamometer MiniDyn 9256C2 together with a digital camera is employed, respectively, for recording the forces and aligning the tool positions.

The work material is supplied in the form of a cylindrical bar with three inches in diameter. The experiments include full immersion slot milling as illustrated in Fig.5. Before conducting experiments, the top surface of the work sample is well prepared with a $\varnothing 2 \mathrm{~mm}$ diameter end mill. For each testing conditions, a total cutting length of $400 \mathrm{~mm}(40 \mathrm{~mm} \times 10$ slots) and $0.5 \mathrm{~mm}$ in width is milled along $-\mathrm{X}$ direction and the gap between adjacent parallel slots is $1.5 \mathrm{~mm}$. The work material is chosen as Al 6061-T6 not only because it is suitable for various engineering applications but also no chemical affinity problems occur against the diamond coatings. The most important is that, due to ductility of the aluminium alloy, antiadhering properties of coatings can be investigated.

The machining conditions used are shown in Table 1 . The sequence of tests is dry cutting using an uncoated tungsten carbide tool, the DLC coated tool, and wet cutting using an uncoated tool. The process parameters, including 60,000rpm spindle speed, 30 $\mu \mathrm{m}$ depth of cut and $60 \mathrm{~mm} / \mathrm{min}$ feed rate, are kept constant under all investigated cutting conditions. In wet cutting at the micro-scale, the forces resulting from the coolant flowing effect becomes comparable to the actual cutting forces and it cannot be neglected. To eliminate this influence on the micro force measurement, cutting fluid is sprayed and accumulates on the top surface of the workpiece beforehand in wet cutting conditions.

\section{Results and discussions on the micro milling performance}

In this section, the experimental results and the associated discussions on the tooling performance are presented. The cutting forces obtained from different cutting conditions are 
firstly discussed, followed by the micro tool integrity, surface roughness and micro-burr formation.

\subsection{Cutting forces and variations with the tool wear progression}

Elementary characteristics for the description of the cutting process are the tool-work interactions. Cutting forces contain important information on the mechanics and dynamics of machining processes and the forces encountered for a tool to cut through a material is directly related to the power consumed. Comparisons of the cutting forces under three cutting conditions are given in Fig.6, including wet cutting using an uncoated tungsten carbide tool (wet WC), dry cutting using an uncoated tungsten carbide (dry WC) and the DLC coated (dry DLC) tools. It is worth to note that only the planar cutting force components are considered in the study. The forces in micro cutting the beginning of each channel are measured to understand an overall perspective of the force variations with the tool wear progression.

At machining the first channel, the feed forces and normal forces show a slight improvement for the DLC coated tool compared with the uncoated tool under dry cutting conditions. However, the values are still dramatically larger than those in wet WC, as clearly seen in Fig.6 (a). The resultant forces at machining the beginning of each slot are plotted in Fig. 6 (b), and it is obviously seen that dry WC has the largest forces, followed by dry DLC, and wet WC has the least. The DLC coated micro tools can reduce around $16 \%$ forces when compared to the uncoated tool in dry cutting conditions. In addition, the forces increase steadily along with the tool wear progression under dry WC and DLC conditions whereas those from wet WC nearly remain constant, which implies that the tool wear for wet WC is the least.

\subsection{Micro tool integrity}

After $400 \mathrm{~mm}$ length cutting, the three micro tools are ultrasonic cleaned and further examined by a digital microscope Keyence VHX-1000 and the tool images are shown in Fig.7. From the bottom view, it is clearly seen that the cutting tips of tools become blunt but no premature failures or breakages of the tool shafts are present.

For the dry WC tool, large-sized built up edges seize close to the two cutting tips, owing to low elastic modulus and ductility of the work material. However, a significant alleviation is found in wet cutting, as shown in Fig.7 (b). Therefore, the use of cutting fluid makes substantial improvement with regard to less adhering. From Fig.7 (c), the DLC coated tool experiences large delaminating around the two cutting tips and the work material aluminium adheres to almost all the delaminating zones. The adhesion is found to be slightly smaller compared to that of the uncoated tool in dry cutting but considerably larger when compared to that in wet cutting. 
The tool flank wear characterization together with corresponding values under various conditions is given in Fig.8. The flank wear is measured at the bottom surface of the micro tool, and the magnitude can be expressed as:

$$
h_{f}=h_{t}-h_{0}
$$

Where $h_{f}-$ The tool flank wear;

$h_{t}$ - Distance between wear point and reference line of a tool after cutting;

$h_{0}$ - Distance between tip point and reference line of a tool before cutting.

The wear on the two tips are averaged and compared in Fig.8 (b) and the values are typically in a few tens of microns under selected cutting conditions. The uncoated tool in dry cutting wears the fastest to around $60 \mu \mathrm{m}$, and the DLC coatings slow down the tool wear by approximate $17 \%$. However, these distinct advantages resulting from the coatings cannot be comparable to that from cutting fluid as wet WC has the lowest flank wear, which is roughly half of that in dry WC conditions. Therefore, wet WC offers the best performance in reducing the tool flank wear, followed by dry DLC, and dry WC.

\subsection{Surface roughness variations with the tool wear progression}

Surface roughness is a measurable surface characteristic quantifying high frequency deviations from an ideal surface. The measurement with assistance of aforementioned white light interferometer is carried out for quantitatively determining the surface roughness variations with the tool wear progression. The values under different cutting conditions are recorded at the beginning and in the middle of each micro milled channel on the bottoms, and they are plotted as a function of cutting distance, as depicted in Fig.9, in which corresponding exponential trend lines are also given.

It is obviously seen that the DLC coated micro tool yields fairly better surface roughness than the uncoated tool in dry cutting but slightly poorer than the uncoated tool in wet cutting. Meanwhile, the band widths of surface roughness variations are greatly reduced when applying the DLC coatings and cutting fluid on micro tools. It is also found that surface roughness under the three cutting conditions has similar trend as the resultant cutting forces do along with the tool wear progression, i.e. the longer cutting length, the worse machined surface quality under dry DLC and WC conditions, and there are no obvious variations for wet cutting using the uncoated tool.

\subsection{Micro-burr formation}

In micro-scale milling, burr formation is inevitable. The presence of micro burrs not only reduces part accuracy and quality but also affects its assembly and functionality. Burrs can be classified as entrance, exit, top and bottom burrs according to the position as well as primary, needle-like, feathery and minor burrs according to the shape and amount [13]. Top burrs on the micro milled channels are examined to further evaluate the tools' performance. The burrs 
from the beginning of each first slot under different cutting conditions are inspected and photographed, and examples are shown in Fig.10.

The burrs are found to be considerably larger on the top entrance side and almost free on the top exit side under all investigated conditions. Generally these visible burrs are in the feathery and wavy type. The DLC coated tool in dry cutting and the uncoated tool in wet cutting produce roughly the same and the smallest burrs, but the uncoated tool in dry cutting the biggest. Thus, the use of DLC coatings and cutting fluid on micro tools has the equal effects in reducing the micro-burr formation.

\section{Conclusions}

In this paper, DLC coatings have been successfully deposited on a $\phi 500 \mu \mathrm{m}$ diameter micro end mill by a PE-CVD process. In order to evaluate the coating performance, coating characterization has been undertaken and full immersion micro milling trials using the DLC coated tool have been performed in dry cutting conditions. The corresponding tooling performance, including the cutting forces, tool integrity, surface roughness and micro-burr formation, have been compared with those under uncoated WC tools in both dry and wet cutting conditions for the assessment. The following conclusions can be drawn:

(1) The preliminary characterization shows the DLC coatings cover well on the micro tool except for a slight delaminating on the edges and the coating surface is considerably smooth.

(2) Roughly $16 \%$ of the force reduction benefits from the DLC coatings. The performance improvement is not comparable with that of cutting fluid, which significantly reduces the forces.

(3) The DLC coated tool experiences delaminating on the two tips and there are large portions of aluminium covering on the delaminating area.

(4) The DLC coatings help reduce approximately $17 \%$ of the flank wear in dry cutting conditions and the wear reduction is still unreachable to that using cutting fluid.

(5) Dry DLC produces considerably better surface roughness than dry WC, whereas slightly worse than wet WC.

(6) Applying DLC coatings and cutting fluid on micro tools fairly minimize the microburr formation and they have almost the equal influence.

Based on above conclusions, major improvements such as reduced cutting forces, slightly less chip adhesion, lower tool flank wear and improved surface roughness as well as smaller micro-burr size can be achieved when applying DLC coatings on the micro end mill, although the positive impact of the coatings is still weaker than that of cutting fluid. However, because of important environmental and economic benefits for dry cutting, it is more desirable and promising to apply DLC coatings rather than cutting fluid on micro tools in promoting the tooling performance for industrial applications. Due to the DLC coating 
delaminating problems, further studies need to be done to optimize the coating conditions so as to enhance bonding strength upon tungsten carbide to meet sustainable manufacturing requirements.

\section{Acknowledgments}

The authors gratefully acknowledge the funding for this research by the UK Technology Strategy Board (TSB) (Grant contract number: BD266E) and Kistler UK Ltd for the dynamometer kit support.

\section{References}

1. Childs T.H.C., Maekawa K., Obikawa T. and Yamane Y. Metal machining: theory and applications, Arnold, London, 2000

2. Wu T., Cheng K. An investigation on the cutting performance of nano-crystalline diamond coatings on a micro-end mill, Proceedings of the Institution of Mechanical Engineers, Part B: Journal of Engineering Manufacture 2012 226, 8, 1421-1424

3. Heaney P.J., Sumant A.V., Torres C.D., Carpick R.W., Pfefferkorn F.E. Diamond coatings for micro end mills: Enabling the dry machining of aluminium at the micro-scale, Diamond and Related Materials 2008 17, 223-233

4. Aramcharoen A., Mativenga P.T. and Yang S. The effect of AlCrTiN coatings on product quality in micro-milling of 45 HRC hardened H13 die steel, Proceedings of the 35th International MATADOR Conference 2007 203-206

5. Aramcharoen A., Mativenga P.T., Yang S., Cooke, K.E. and Teer, D.G. Evaluation and selection of hard coatings for micro milling of hardened tool steel, International Journal of Machine Tools and Manufacture 2008 48, 14, 1578-1584

6. Union Tool, Available at: http://www.uniontool.co.jp/ (Accessed: 8/13/2012)

7. Torres C.D., Heaney P.J., Sumant A.V. Hamilton M.A., Carpick R.W., Pfefferkorn F.E. Analyzing the performance of diamond-coated micro end mills, International Journal of Machine Tools and Manufacture 2009 49, 599-612

8. Dai M., Zhou K., Yuan Z., Ding Q., Fu Z. The cutting performance of diamond and DLCcoated cutting tools, Diamond and Related Materials 2000 9, 1753-1757

9. Fukui H., Okida J., Omori N., Moriguchi H., Tsuda K. Cutting performance of DLC coated tools in dry machining aluminium alloys, Surface and Coatings Technology 2004 $187,70-76$

10. Hanyu H., Kamiya S., Murakami Y., Kondoh Y. The improvement of cutting performance in semi-dry condition by the combination of DLC coating and CVD smooth surface diamond coating, Surface and Coatings Technology 2005 200, 1137-1141 
11. Wu T., Cheng K. 3D FE-based modelling and simulation of the micro milling process, Proceedings of Precision Engineering and Nanotechnology, Key Engineering Materials 2012 516, 634-639

12. Wu T., Cheng K., Rakowski R. Investigation on tooling geometrical effects of micro tools and the associated micro milling performance, Proceedings of the Institution of Mechanical Engineers, Part B: Journal of Engineering Manufacture 2012 226, 9, 1442 1453

13. Chern G.L., Wu Y.J.E., Cheng J.C., Yao J.C. Study on burr formation in micromachining using micro-tools fabricated by micro-EDM, Precision Engineering 2007 31, $122-129$ 


\section{List of figure captions:}

Fig.1 SEM images of the uncoated micro tool

Fig.2 The PE-CVD reactor and system

Fig.3 Integrity of the DLC coated micro tool

Fig.4 Measurement of surface roughness on the tool shanks

Fig.5 Schematic of the micro milling experiment

Fig.6 Cutting force comparisons under different cutting conditions

Fig.7 Micro tools after the micro milling tests

Fig.8 Micro tool flank wear characterization and corresponding values

Fig.9 Surface roughness variations under different conditions

Fig.10 Examples of micro-burr formation under different conditions 

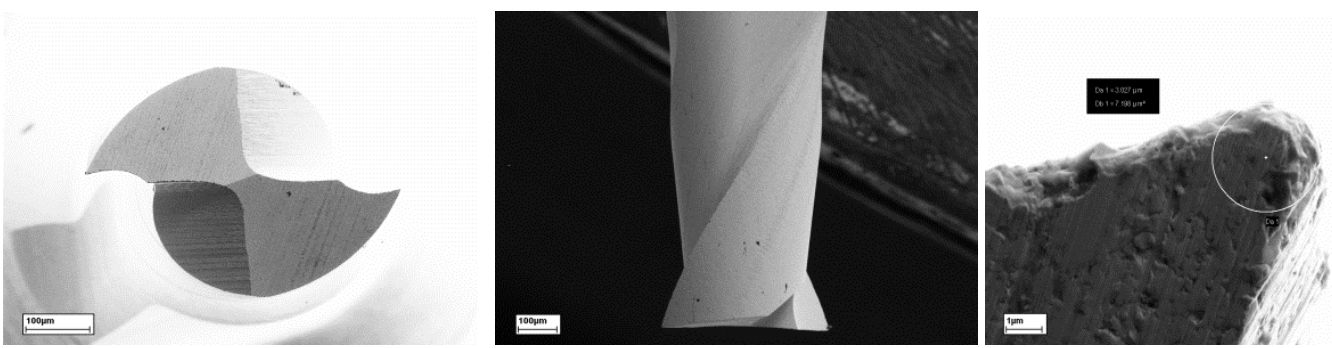

Fig.1 SEM images of the uncoated micro tool

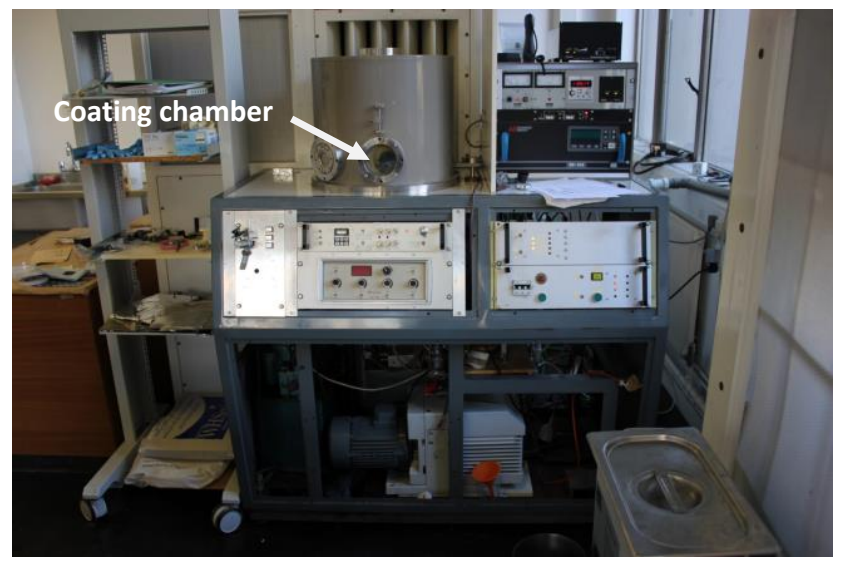

Fig.2 The PE-CVD reactor and system

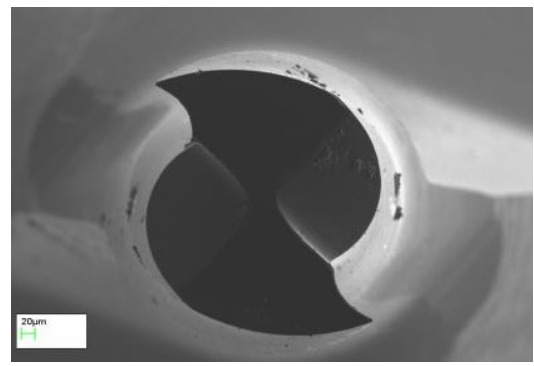

(a) Bottom view

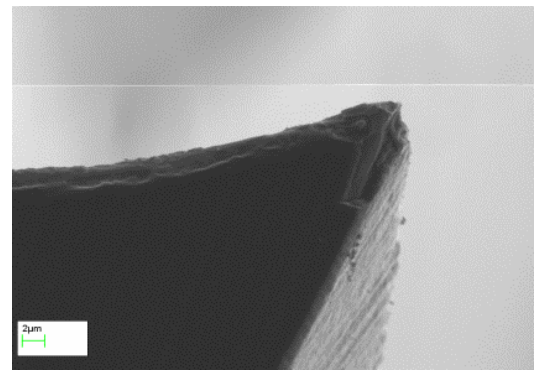

(c) Delaminating on the tip

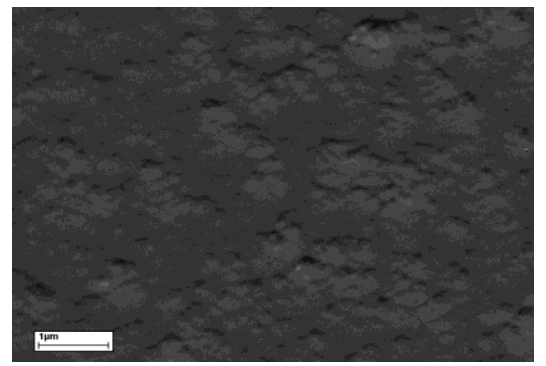

(b) Bottom coating surface

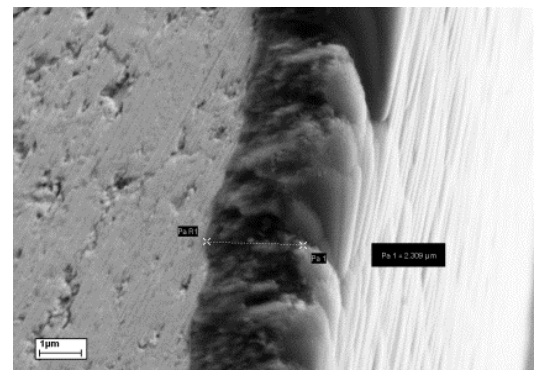

(d) Coating thickness measurement

Fig.3 Integrity of the DLC coated micro tool 


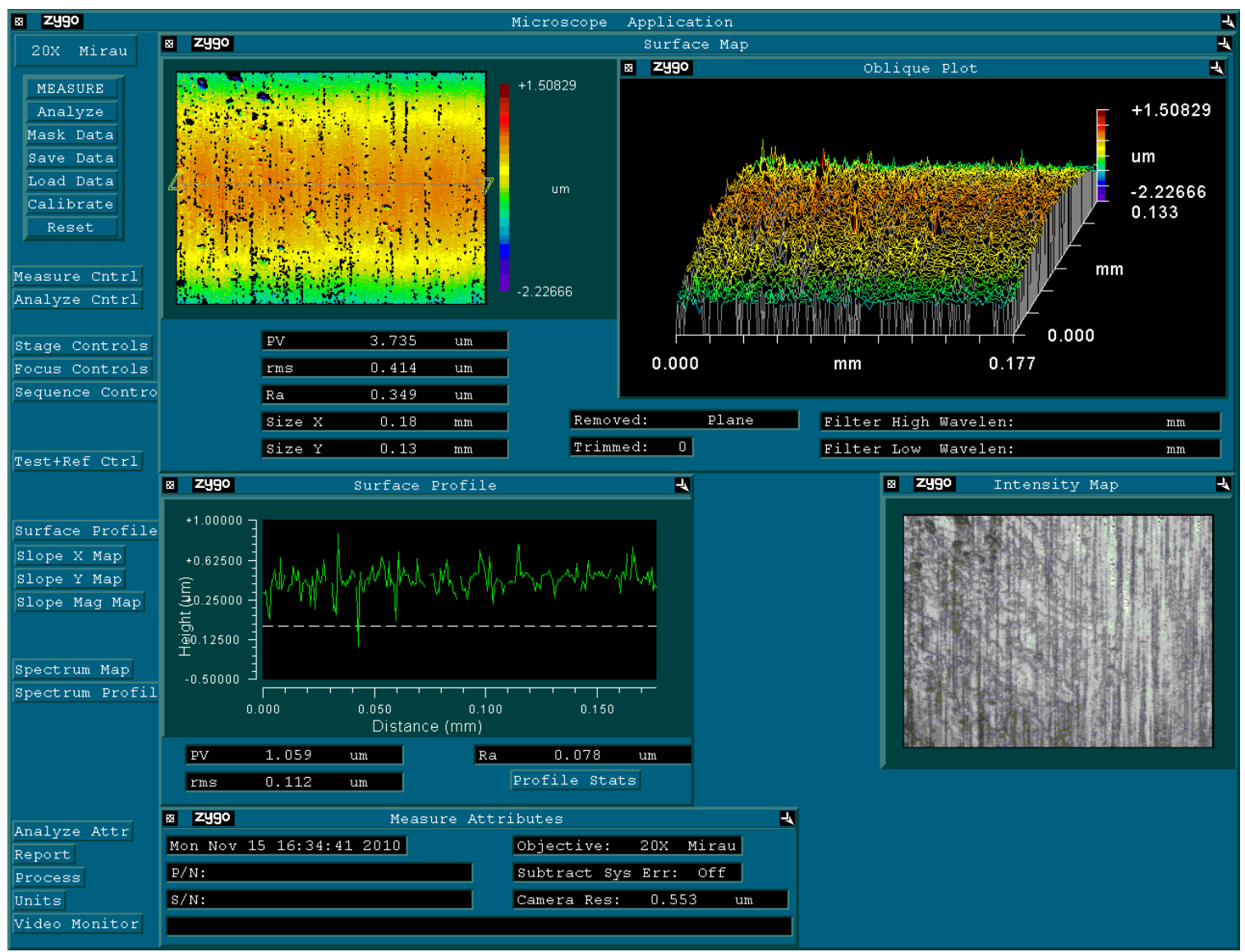

(a) Tungsten carbide tool (78 nm) 


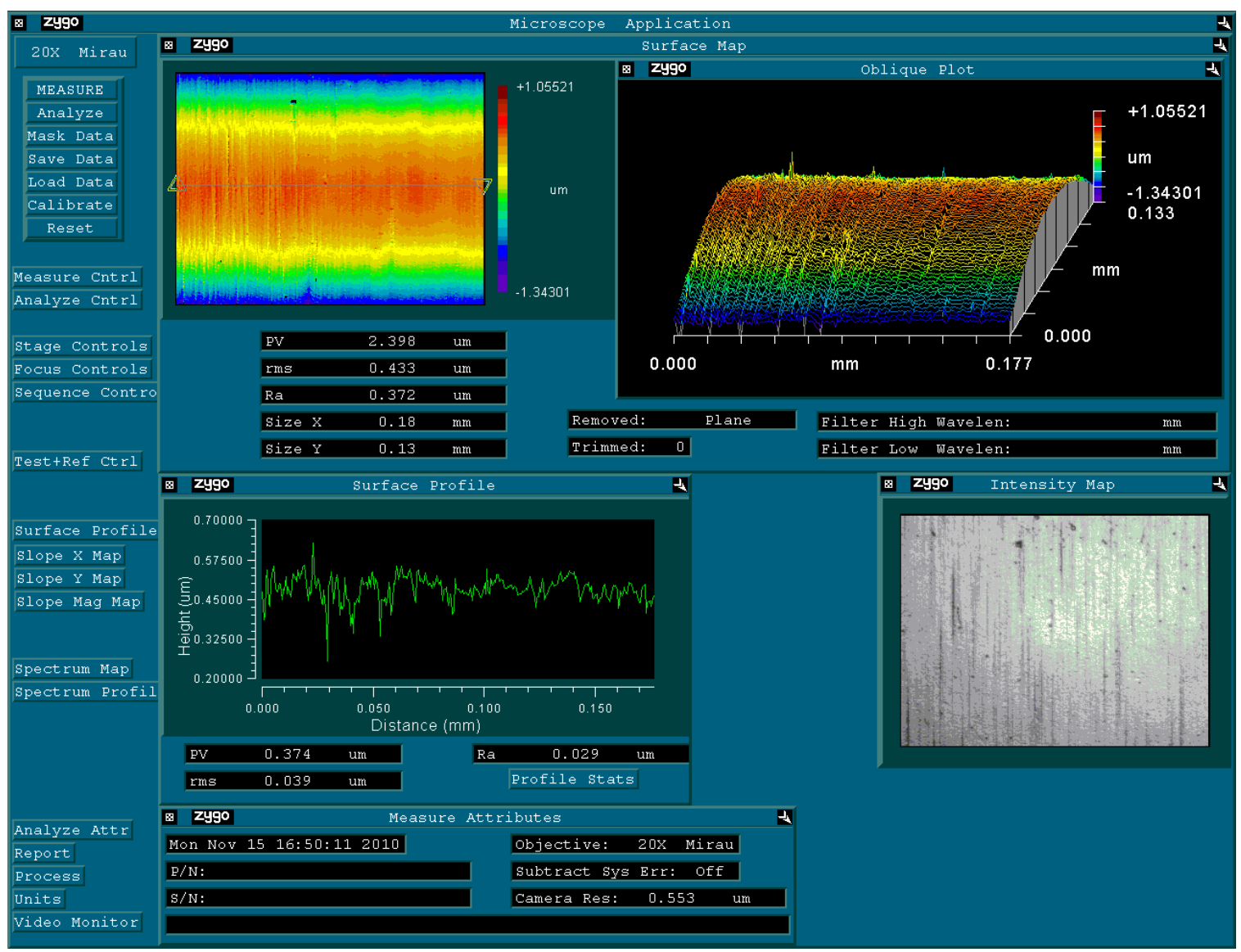

(b) DLC coated tool (29 nm)

Fig.4 Measurement of surface roughness on the tool shanks

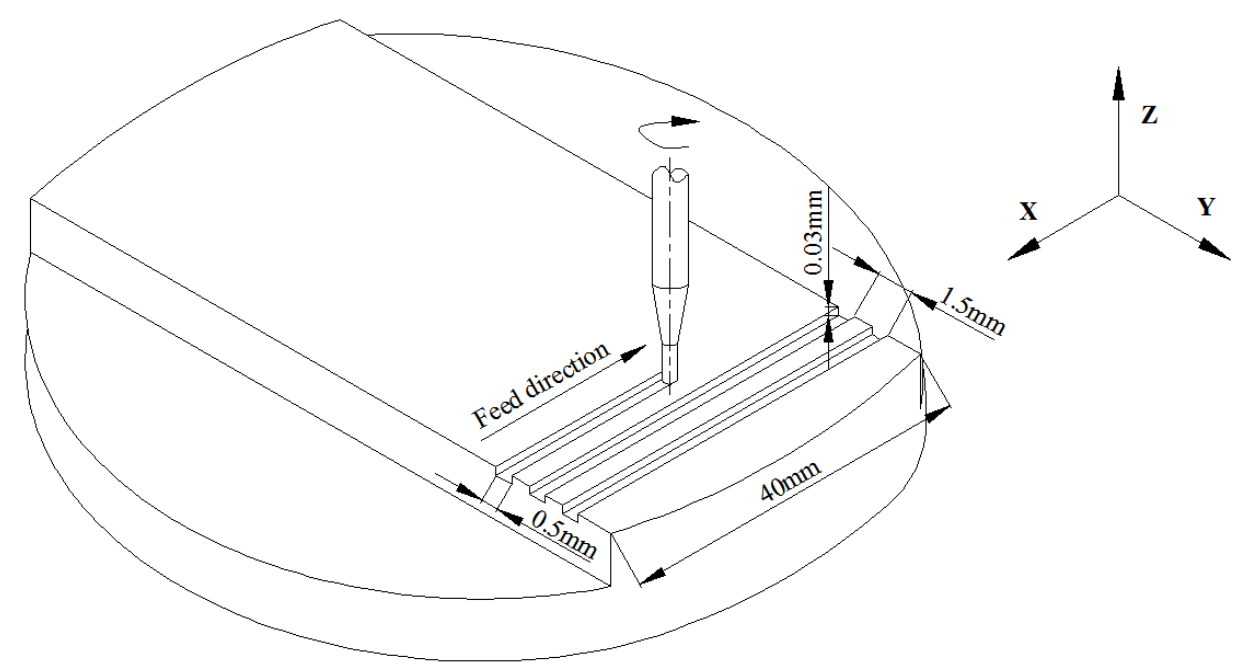

Fig.5 Schematic of the micro milling experiment 

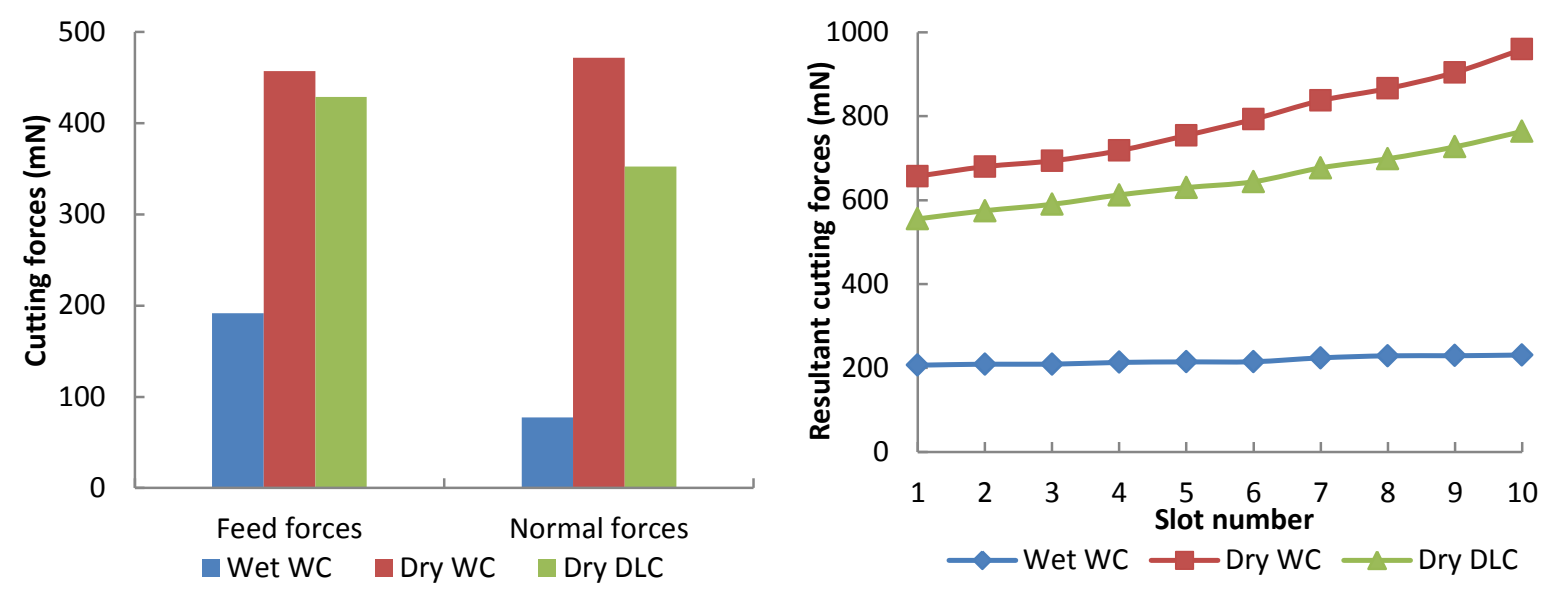

(a) The cutting force components at the first slot (b) The resultant force variations with the tool wear progression

Fig.6 Cutting force comparisons under different cutting conditions

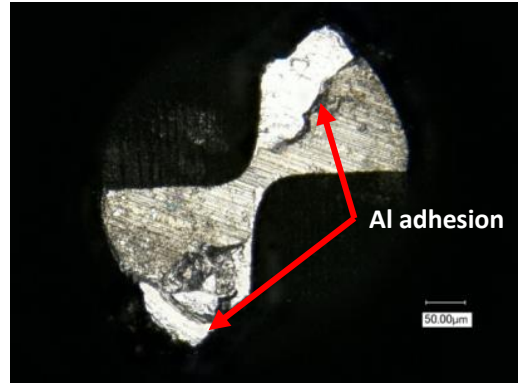

(a) Dry WC

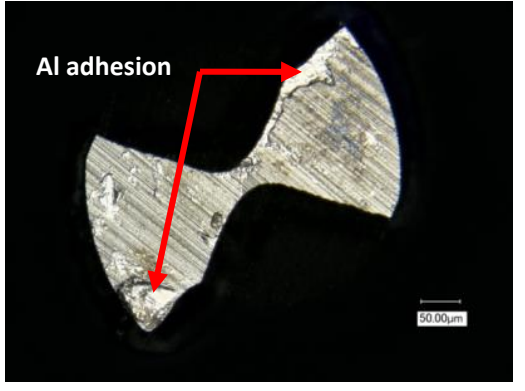

(b) Wet WC

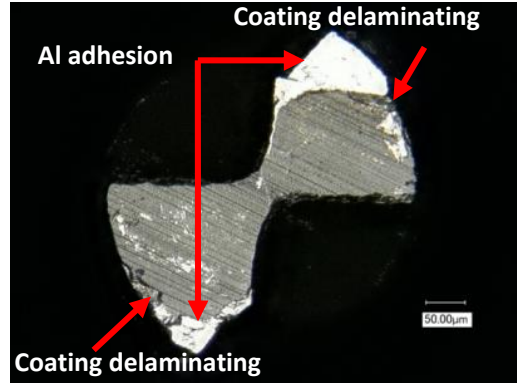

(c) Dry DLC

Fig.7 Micro tools after the micro milling tests 


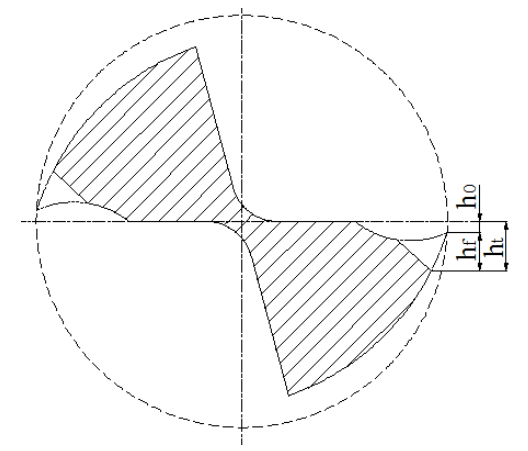

(a) Flank wear characterization

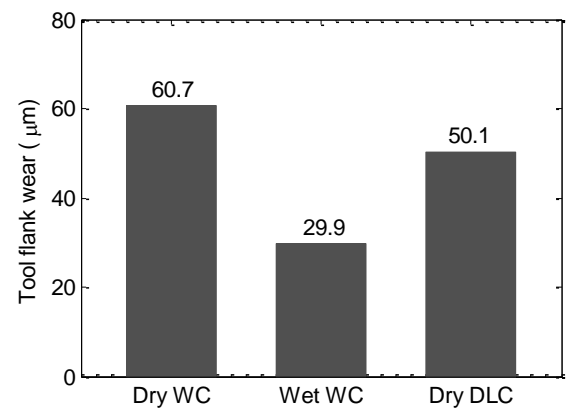

(b) Tool wear under various conditions

Fig.8 Micro tool flank wear characterization and corresponding values

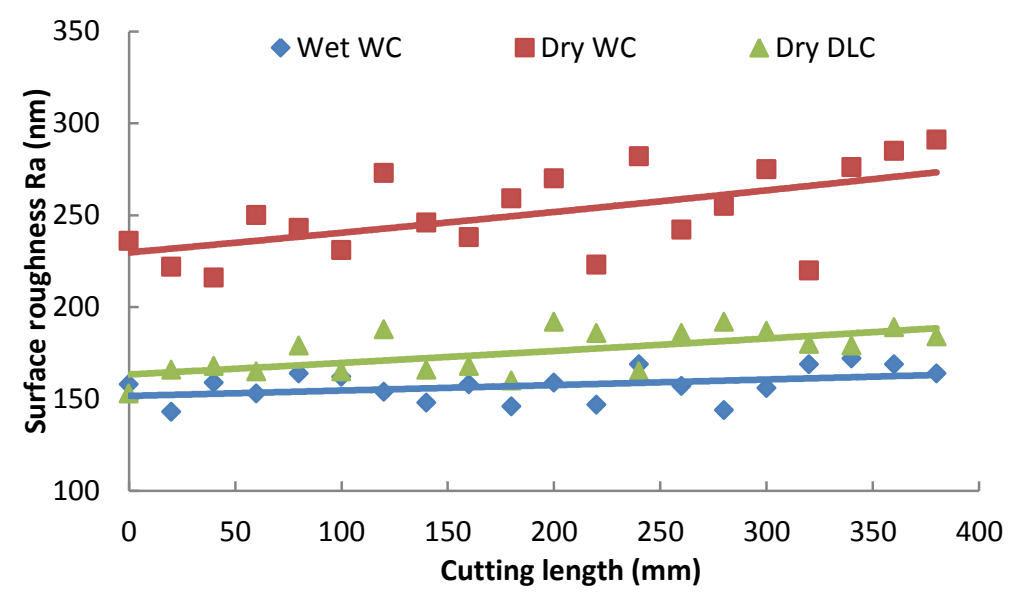

Fig.9 Surface roughness variations under different conditions

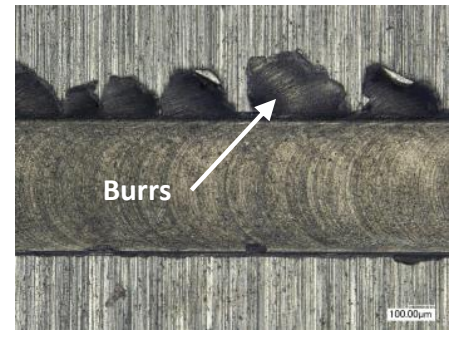

(a) Dry WC

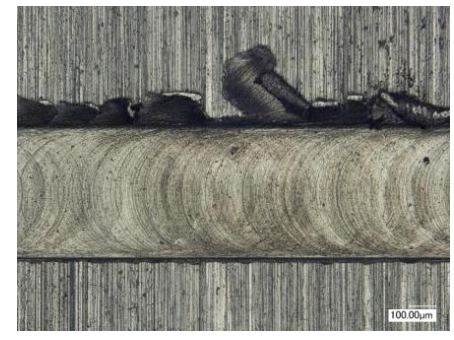

(b) Wet WC

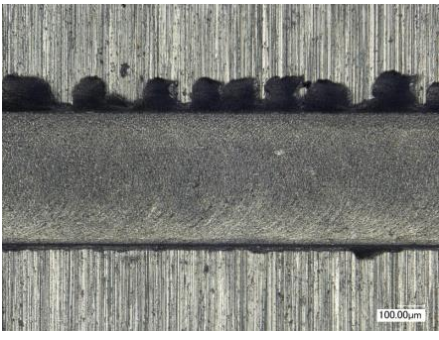

(c) Dry DLC

Fig.10 Examples of micro-burr formation under different conditions 